\title{
XMM-Newton observations of MR Vel/RX J0925.7-4758
}

\author{
C. Motch ${ }^{1}$, H. Bearda ${ }^{2,3}$, and C. Neiner ${ }^{4}$ \\ 1 Observatoire Astronomique, UA 1280 CNRS, 11 rue de l'Université, 67000 Strasbourg, France \\ 2 Astronomical Institute, Utrecht University, PO Box 80000, 3508 TA Utrecht, The Netherlands \\ 3 Terschelling 120, 3524 AZ Utrecht, The Netherlands \\ ${ }^{4}$ Sterrenkundig Instituut Anton Pannekoek, Kruislaan 403, 1098 SJ Amsterdam, The Netherlands
}

Received 21 May 2002 / Accepted 27 June 2002

\begin{abstract}
We report on XMM-Newton observations of the galactic supersoft X-ray source RX J0925.7-4758. The RGS spectrum exhibits a wealth of spectral features from iron and oxygen. XMM-Newton data confirm the finding of previous Chandra HETGS/MEG observations that NLTE models of hot white dwarf atmospheres fail to represent the complex spectrum. There are clear evidences for P Cygni profiles with wind velocities of up to $2000 \mathrm{~km} \mathrm{~s}^{-1}$. Small flux variations with time scales larger than $1000 \mathrm{~s}$ are present. The strongest power is at $\sim 0.21 \mathrm{~d}$, a period close to that seen in $V$ band optical light curves. A detailed analysis of the associated changes in the RGS and EPIC pn spectra hint at a mostly grey mechanism suggesting a variation of the visibility of the white dwarf due to occulting material in the accretion disk. Finally, we detect radial velocity changes of $173 \pm 47 \mathrm{~km} \mathrm{~s}^{-1}$ between two RGS observations obtained half an orbital cycle apart. The amplitude of the RGS velocity shift is consistent with that of the optical He II $\lambda 4686$ and thus supports the idea that most of the He II optical line emission arises from the accretion disk.
\end{abstract}

Key words. X-rays: binaries - X-rays: individual: RX J0925.7-4758 - stars: individual: MR Vel - stars: mass-loss techniques: radial velocities

\section{Introduction}

Supersoft X-ray sources have been discovered by the Einstein satellite and extensively studied by ROSAT. They usually consist of a close binary system in which the accreting component is a very hot white dwarf responsible for the soft thermal-like radiation (van den Heuvel et al. 1992). At the low spectral resolution of the ROSAT PSPC, the energy distributions look like black bodies with temperatures in the range of 20 to $70 \mathrm{eV}$. Higher resolution spectra with ASCA (e.g. Ebisawa et al. 1996) started to reveal complex structures later emphasized by Chandra and XMM-Newton grating spectroscopy (Paerels et al. 2001; Bearda et al. 2002). Fitting model atmospheres of hot white dwarfs usually imply bolometric luminosities below but close to Eddington. Stable or quasi stable hydrogen burning at the surface of the white dwarf can adequately explain most of the observational features of supersoft X-ray sources (van den Heuvel et al. 1992). The high accretion rates required by stable thermonuclear burning $(\dot{M} \sim$ $10^{-7} M_{\odot} \mathrm{yr}^{-1}$, Iben 1982) can be obtained through thermally unstable Roche lobe overflow when the mass donor star is more massive than the white dwarf. However, this picture is probably not valid for many sources since short periods systems cannot accommodate a massive mass-donor star. In these cases, strong irradiation by the very luminous central source

Send offprint requests to: $\mathrm{C}$. Motch, e-mail: motch@astro.u-strasbg.fr could play a major role in driving the mass transfer mechanism (van Teeseling \& King 1998).

Because stable burning allows matter to pile up at the surface of the white dwarf, contrary to novae where most matter is expelled in the runaway event, supersoft sources are considered as possible SN Ia progenitors. A sizeable fraction of SN Ia could occur in these systems when the white dwarf approaches the Chandrasekhar limit (Rappaport et al. 1994).

We report in this paper on a long XMM-Newton observation of RX J0925.7-4758 involving the EPIC pn, RGS and OM instruments. RX J0925.7-4758 is one of the few galactic supersoft source known (Motch et al. 1994). It is identified with a $V \sim 17.2$ reddened object exhibiting Balmer and high excitation lines of He II, $\mathrm{O}$ VI and $\mathrm{C}$ IV. The relatively high interstellar absorption towards the source $\left(N_{\mathrm{H}} \sim 10^{22} \mathrm{~cm}^{-2}\right)$ only allows observation in the highest energy range (above $\sim 0.4 \mathrm{keV}$ ). Among supersoft sources, RX J0925.7-4758 is unusual by its relatively high X-ray temperature and long orbital period of 4.0287 d (Schmidtke \& Cowley 2001). Previous X-ray observations have been reported from a number of satellites (ASCA; Ebisawa et al. 2001, BeppoSAX; Hartmann et al. 1999 and recently using the HETG on board Chandra; Bearda et al. 2002).

\section{XMM-Newton and optical observations}

The XMM-Newton satellite is described in Jansen et al. (2001). XMM-Newton observations took place in late December 2000 
Table 1. Journal of observations.

\begin{tabular}{lc}
\hline \hline \multicolumn{2}{l}{ Rev 187: 2000-12-16 11:01-2000-12-17 04:05 } \\
Photometric phase : $0.82-0.98$ \\
\hline Instrument & Exposure time (s) \\
EPIC MOS 1+2 & 58400 \\
EPIC pn & 56700 \\
RGS 1+2 & 61200 \\
\hline Rev 188: 2000-12-18 10:51-2000-12-19 04:06 \\
Photometric phase : 0.31-0.47 \\
\hline Instrument & Exposure time (s) \\
EPIC MOS 1+2 & 59000 \\
EPIC pn & 57300 \\
RGS 1+2 & 61600 \\
\hline
\end{tabular}

during two consecutive satellite orbits, thus covering two phase intervals of the binary period half a cycle apart. The original scheduling was done using the ephemeris from Schmidtke et al. (2000) which assumed a $3.83 \mathrm{~d}$ period and was planned to cover photometric phases 0.0 (minimum) and 0.5 . With the revised period of $4.0287 \mathrm{~d}$ (Schmidtke \& Cowley 2001) the actual range of phase covered is slightly shifted towards earlier times. The journal of X-ray observations is listed in Table 1. We also organized quasi-simultaneous optical observations using the Dutch telescope on La Silla during one night. In December, RX J0925.7-4758 was only observable in the last part of the night just after the end of the first XMM-Newton observation.

The EPIC background remained nominal during the two observations apart for the last $2000 \mathrm{~s}$ of the second one. EPIC pn data were acquired in the large window mode in order to minimize pile-up problems from this relatively bright source (up to $7 \mathrm{cts} / \mathrm{s}$ in EPIC pn). The MOS was left in imaging mode but the source count rate is too high for proper spectral analysis due to severe pile up effects. The OM was used in normal imaging mode and because of the presence of bright stars in the field of view, the UVW1 filter was used. RGS data had to be completely reprocessed from the ODF late October 2001 when good calibrations at high energies eventually became available. All data have been analyzed using SAS version 20010917_1110.

Optical observations were carried out using the standard CCD camera at the Dutch telescope located on ESO La Silla, Chile. $V$ and $B$ filters were alternated with exposure times of 3 and $5 \mathrm{~min}$ in the $V$ and $B$ filters respectively. Standard MIDAS procedures were used to correct images for flat-field and bias. Instrumental magnitudes were computed using the Sextractor package (Bertin \& Arnouts 1996) and linked to comparison star 2 in Motch et al. (1994).

\section{Source variability}

Figure 1 shows the simultaneous EPIC pn and OM UVW1 and the contemporaneous $V$ band light curve obtained at the Dutch telescope. The EPIC pn light curve displays significant variability at the level of ten percent on various time scales. Some kind of oscillation with a period of $\sim 0.2 \mathrm{~d}$ is clearly visible as well as faster variability. Not surprisingly considering the high
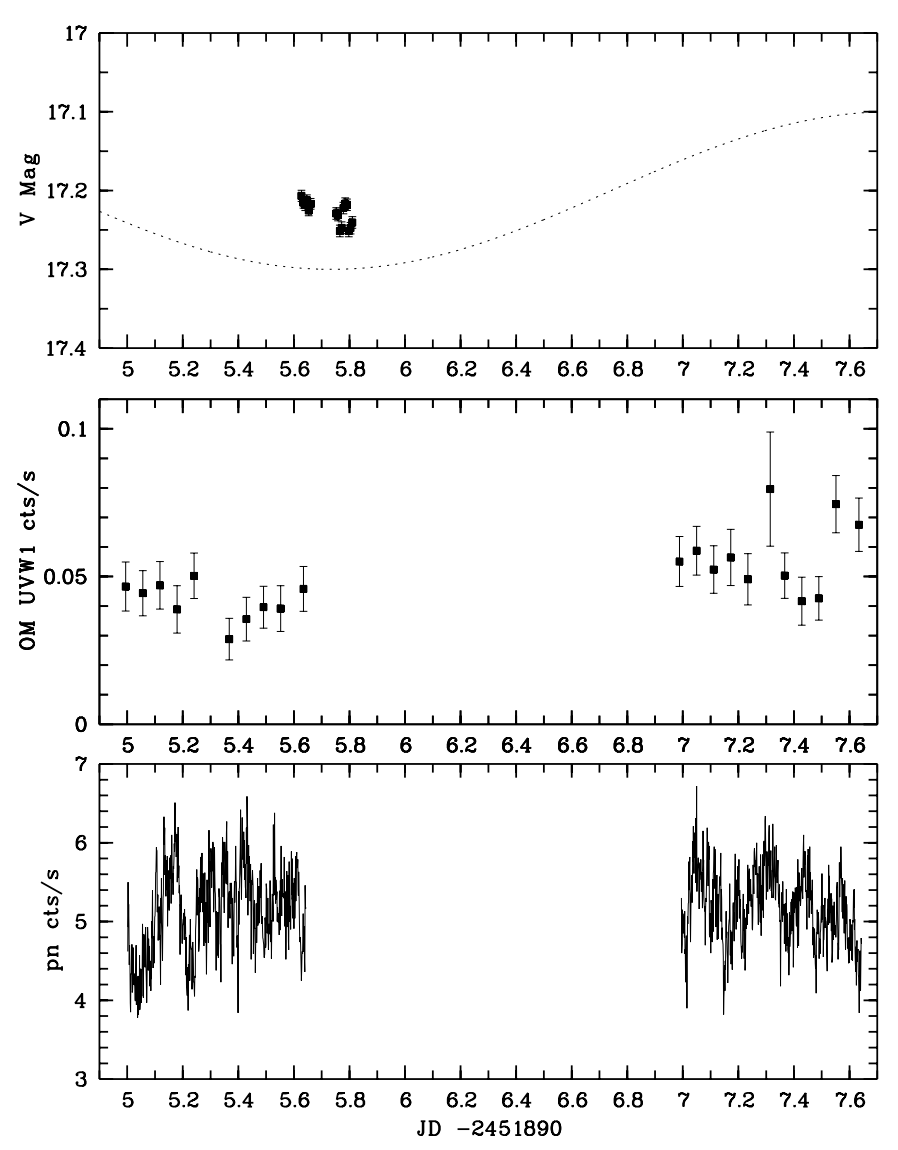

Fig. 1. Simultaneous EPIC pn (0.5-1.0 keV, $100 \mathrm{~s}$ bins), OM and $V$ band Dutch data. The dotted line in the upper panel represents the mean photometric V light curve taken from Schmidtke et al. (2000) assuming the revised orbital period of $4.0287 \mathrm{~d}$.

interstellar absorption towards the source $\left(N_{\mathrm{H}} \sim 10^{22} \mathrm{~cm}^{-2}\right)$ OM UVW1 data are of much lower statistical quality, each point representing an integration over $5000 \mathrm{~s}$. There is no compelling evidence for any strong correlations between X-ray and UV flux variations. During the XMM-Newton observations, the mean $V$ and $B$ magnitudes are similar to those usually observed $(\langle V\rangle=17.23$ and $\langle B-V\rangle=1.88$ ) indicating that RX J0925.7-4758 was not in an especially bright or low optical state. The $V$ level is only $\sim 0.07$ mag above the mean value from Schmidtke et al. (2000), consistent with the observed cycle to cycle intrinsic variability.

We searched for coherent oscillations in each individual observation as well as in the combined data sets using the least square power spectrum method outlined in Lomb (1976) and Scargle (1982). Light curves with bin sizes of 10 and $100 \mathrm{~s}$ $(0.5-1.0 \mathrm{keV})$ do not show any statistically significant pulsations for periods in the range of 30 to $1000 \mathrm{~s}$. There is however a clear power excess at low frequencies on time scales larger than $1000 \mathrm{~s}$ which can be seen in the detailed light curve (Fig. 2) and in the power spectrum of the combined EPIC pn observations (see Fig. 3). The highest peak in the periodogram of each individual observation as well as in that of the merged EPIC pn light curves is at $P \sim 0.21 \mathrm{~d}$. Its formal statistical significance is high and the modulation is well seen in Fig. 1. Best periods in revolutions 187 and 188 are $P=0.2152 \pm 0.0059 \mathrm{~d}$ and 

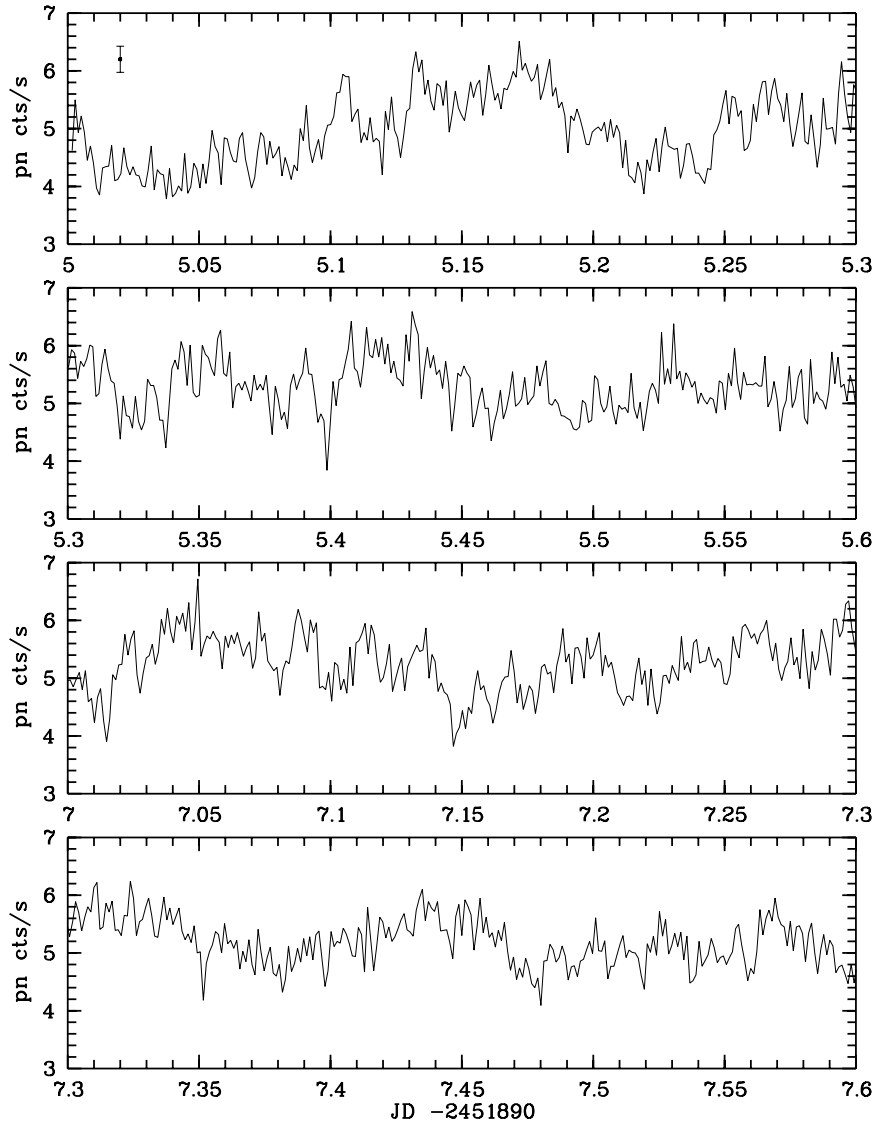

Fig. 2. Details of the EPIC pn light curve (0.5-1.0 keV, $100 \mathrm{~s}$ bins). A typical error bar is shown in the upper panel. Variations on time scales larger than $\sim 1000 \mathrm{~s}$ are clearly seen in the power spectrum.

$P=0.1977 \pm 0.0082 \mathrm{~d}$ respectively and are therefore consistent with a constant value. The periodogram of the merged EPIC pn data has its highest peak at $P=0.2130 \pm 0.009 \mathrm{~d}$. Observations over many more cycles would be needed to be able to measure the stability of this X-ray modulation and especially compare it with that of the $0.23 \mathrm{~d}$ quasi-periodicity detected in the optical by Schmidtke et al. (2000). The mean full amplitude of the best fit sine wave to the $0.2 \mathrm{~d}$ modulation is $10.8 \%$.

\section{RGS spectroscopy}

\subsection{Overall spectrum}

Thanks to the high throughput of the RGS and long total exposure time $(\sim 120 \mathrm{ks})$, the spectrum of RX J0925.7-4758 summed over the two observations and the two RGS reveals an unprecedented number of spectral features compared to the Chandra HETG spectrum discussed in Bearda et al. (2002). We show in Fig. 4 the total XMM-Newton spectrum together with tentative line identifications. This spectrum was accumulated using SAS task rgsfluxer and the corresponding reprocessed extractions and response matrices computed for each individual instrument and observation. Spectral bins with large associated errors have been removed from the plot for clarity. All features identified in the Chandra spectrum can be seen in the RGS data. The spectrum is clearly not black body like and exhibits a large

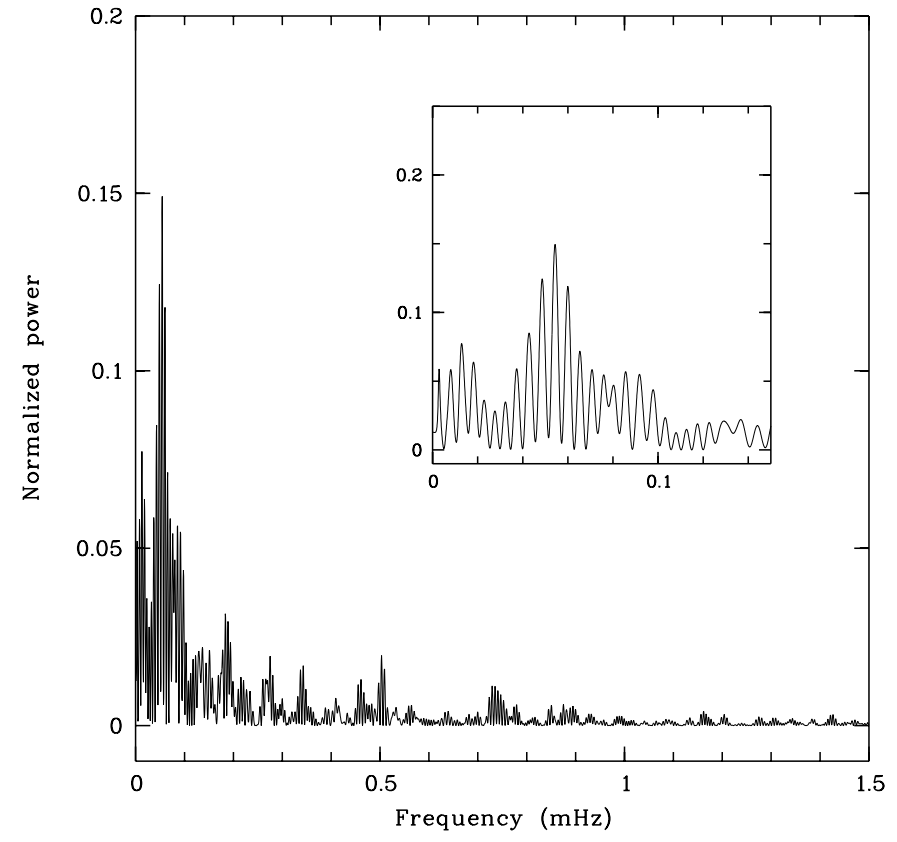

Fig. 3. Power spectrum of the combined EPIC pn light curve of the two observations $(0.5-1.0 \mathrm{keV})$. The highest peak is at $P \sim 0.21 \mathrm{~d}$, a period close to that reported for the optical quasi-oscillations by Schmidtke et al. (2000).

number of high excitation iron lines, mostly seen in emission as well as lines of the $\mathrm{O}$ vIII Lyman series. The conclusions of Bearda et al. (2002) that the structures seen in the Chandra spectrum cannot be reproduced by LTE or NLTE model atmospheres can only be emphasized by our RGS data. Figure 5 shows the "best" fit NLTE white dwarf model atmosphere of Bearda et al. (2002) which has $\log g=9$ and $T=8.9 \times 10^{5} \mathrm{~K}$. The intensity of the RGS spectrum in the $\mathrm{O}$ edge region suggests an absorption of $N_{\mathrm{H}}=1.50 \times 10^{22} \mathrm{~cm}^{-2}$ slightly higher than that assumed by Bearda et al. (2002) for HETGS/MEG data $\left(N_{\mathrm{H}}=1.0 \times 10^{22} \mathrm{~cm}^{-2}\right)$. However, ignoring this long wavelength region, a better fit is obtained in the 17 to $20 \AA$ range with $N_{\mathrm{H}}=1.28 \times 10^{22} \mathrm{~cm}^{-2}$. Overall, the picture is similar to that drawn from Chandra data, namely that although the shape of the energy distribution seems to be grossly described by the model, strong emission features such as the $\mathrm{O}$ vIII lines or the Fe xvII lines at $\lambda 15.015 \AA$ and $\lambda 15.262 \AA$ are not present in the model atmosphere. We did not investigate further spectral modelling, leaving this study open for future work. The lack of $\mathrm{X}$-ray flux in the RGS and EPIC below $\sim 0.4 \mathrm{keV}$ argues for a strong interstellar absorption consistent with that seen at optical wavelengths $\left(N_{\mathrm{H}} \sim 10^{22} \mathrm{~cm}^{-2}\right)$. Interstellar $\mathrm{O}$ edge is well marked at $23.3 \AA$ and there are clear evidences for the presence of Fe-L and Ne edges at $17.54 \AA$ and $14.30 \AA$ respectively, although, part of these structures could also be due to intrinsic spectral features from the hot atmosphere.

In the absence of proper modelling of the emission spectrum it is impossible to derive fundamental parameters such as temperature, density, gravity and luminosity. Also, since most of the lines are suspected to be blended, line profile distortions due to bulk motion for instance are difficult to analyse in details. There seems however to be a clean case of a P Cygni 


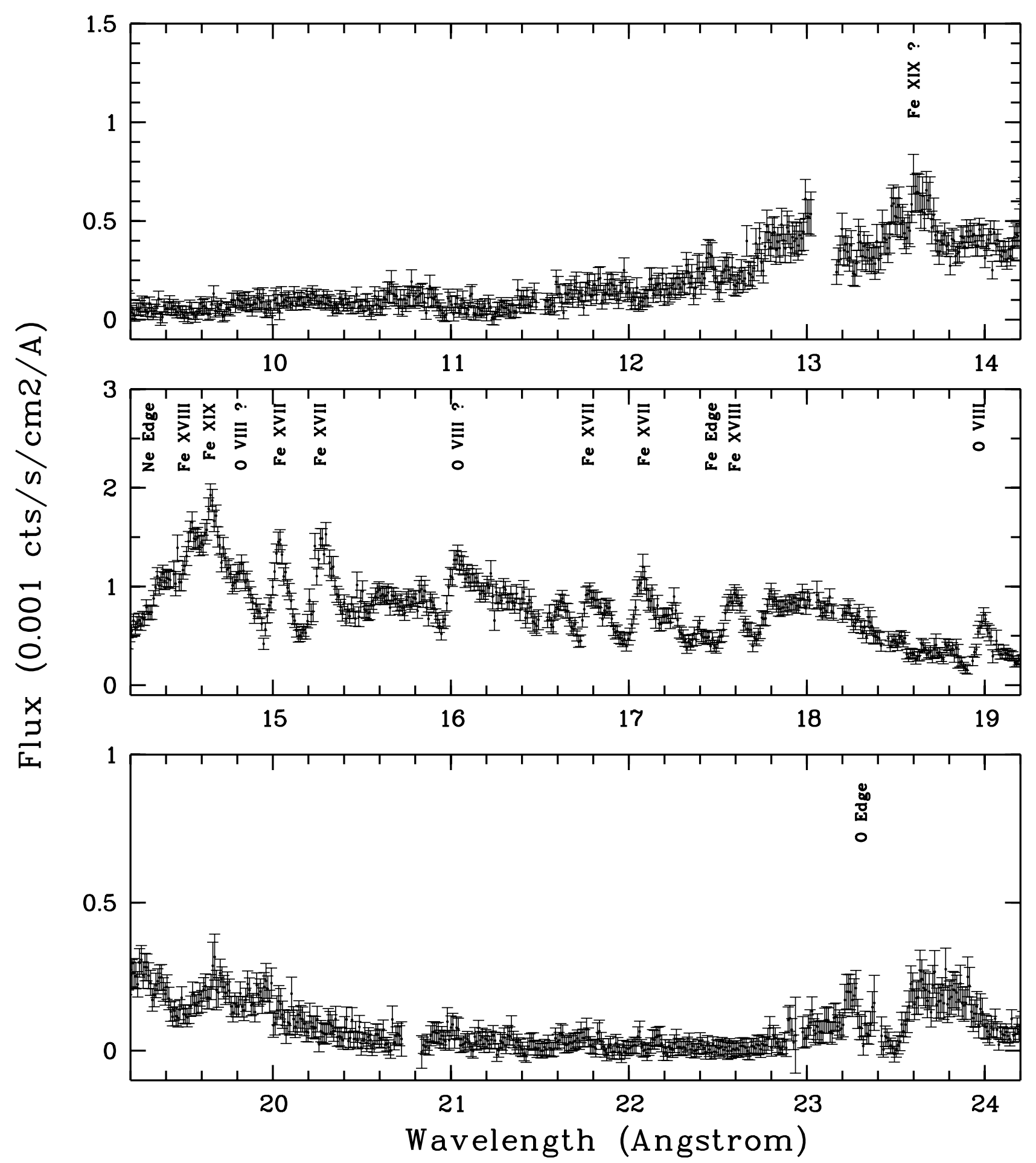

Fig. 4. The RGS spectrum of RX J0925.7-4758 summed over the two observations and the two RGS instruments and corrected for effective area.

profile in the Lyman $\alpha$ O viII line at $18.97 \AA$, which is relatively free of contaminating lines from other species. As noted by Bearda et al. (2002), other Fe lines have P Cygni - like profiles as well as the probable identification of Lyman $\beta \mathrm{O}$ VIII line at $16.00 \AA$ which is however, suspected of being blended with Fe lines. The blue edge of the mean Ly $\alpha \mathrm{O}$ vIII P Cygni profile shown in Fig. 7 is at velocities close to $2000 \mathrm{~km} \mathrm{~s}^{-1}$ slightly larger than the $1500 \mathrm{~km} \mathrm{~s}^{-1}$ derived by Bearda et al. (2002), but much less than that of the transient collimated wind or jet discovered by Motch (1998).

\subsection{Variability with orbital phases and flux level}

The RGS 1 and 2 summed spectra for revolutions 187 and 188 are identical within the error bars. Over the 13 to $20 \AA$ range 

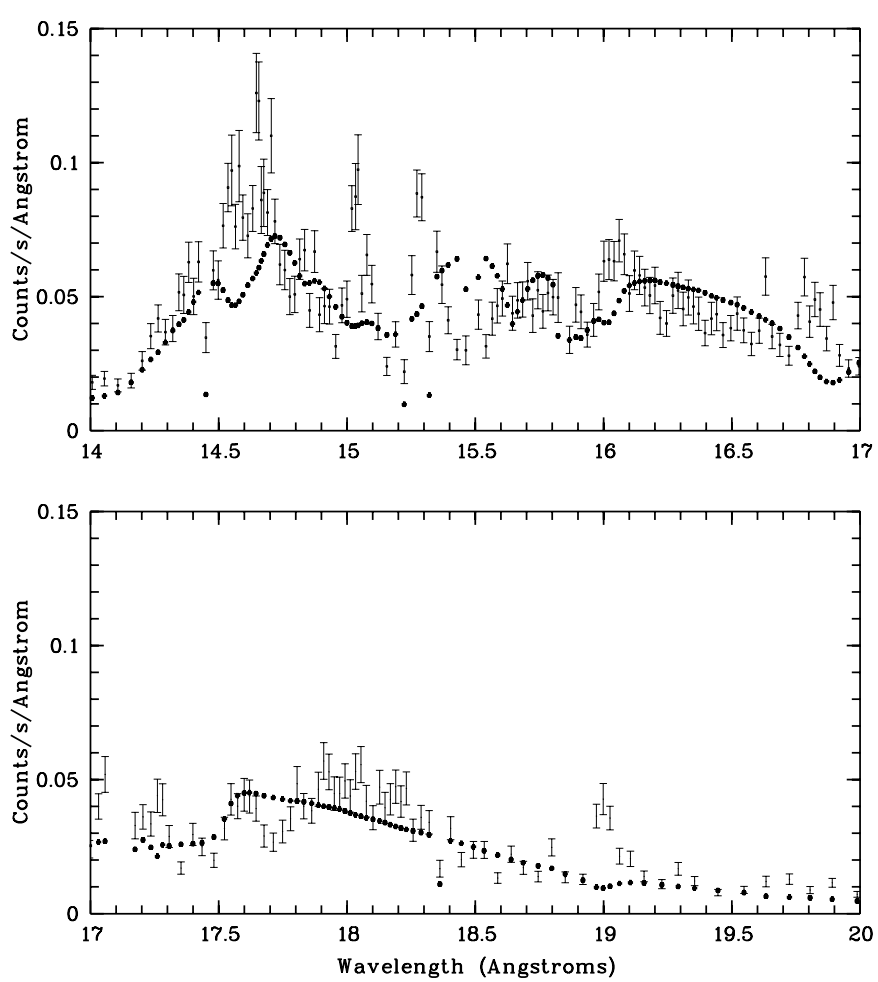

Fig. 5. The RGS - 1 spectrum of RX J0925.7-4758 summed over the first observation. The "best" fit hot white dwarf NLTE model atmosphere of Bearda et al. (2002) with $N_{\mathrm{H}}=1.28 \times 10^{22} \mathrm{~cm}^{-2}$ is shown for comparison.

the source appears slightly brighter by $\sim 12 \pm 4 \%$ in the last observation. However, the flux increase seems to occur over the whole energy range and the difference RGS spectrum does not show marked features which would give hints towards a change in the physical conditions of the source or of its environment.

The variability seen in the EPIC light curve on time scales larger than $1000 \mathrm{~s}$ is also detected in the RGS light curves. We thus tried to see whether marked differences could be found in the line intensities of the low and high flux states in order to shed light on the mechanism giving rise to the small flux variations. For that purpose, we created RGS light curves by selecting events in the area of the $\beta / x$-disp RGS plane where the source lies (see den Herder et al. 2001 for a description of the RGS on board XMM-Newton). For each observation, a summed RGS 1 and 2 light curve was created with $100 \mathrm{~s}$ bins. Two time intervals series corresponding to count rates below and above $0.73 \mathrm{cts} / \mathrm{s}$ and $0.61 \mathrm{cts} / \mathrm{s}$ for the first and second observations respectively were used to accumulate low and high state RGS spectra with similar exposure times. The last part of the second observation, contaminated by high background has been discarded. SAS task rgsfluxer was then used to add together low and high state spectra of different instruments and observations. Overall the high to low count rate ratio is 1.23 . The two spectra shown in Fig. 6 are very similar. The only noticeable differences are a possible higher emissivity at $\sim 13.62 \AA$ (Fe xIX?), 16.05 (Fe xVIII, O vIII?) and $17.40 \AA$ and lower emissivity at $\sim 14.44 \AA, 14.52 \AA$ (Fe xvIII?) in the high flux spectrum. The Lyman $\alpha \mathrm{O}$ vIII $\mathrm{P}$ Cygni profile, once corrected for the different flux levels shows some evidence

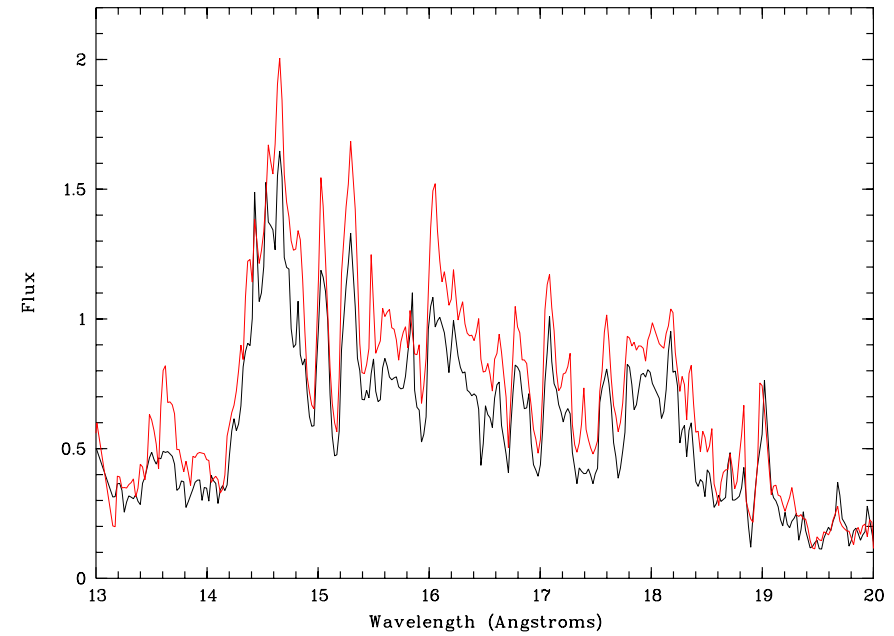

Fig. 6. RGS $1+$ RGS 2 spectra accumulated for the low and high flux states.

for a flux dependence. Although the terminal velocity remains close to the $\sim 2000 \mathrm{~km} \mathrm{~s}^{-1}$ seen in the average profile (Fig. 7), the low velocity part of the profile seems more absorbed (or the emission less strong) at low flux. The two RGS individually give similar pictures, albeit with a lower significance.

We applied the same analysis to EPIC pn spectra, using this time EPIC pn count rates to define three flux levels (high, low and medium) with equal corresponding exposure times. The mean EPIC pn count rate of RX J0925.7-4758 is $\sim 6 \mathrm{cts} / \mathrm{s}$, well below the limit of $12 \mathrm{cts} / \mathrm{s}$ set for pile-up in the large window mode. In order to minimize any residual effect due to pile-up we restricted our analysis to pn pattern $=0$ events. Figure 8 shows the ratio of the high to low EPIC pn spectra plotted for the merged revolutions 187 and 188 data. Although the NLTE model atmosphere of Bearda et al. (2002) does not satisfactorily fit the EPIC pn spectrum, it provides a better representation than black body spectra and can be used to estimate the expected distortion of the energy distribution if it was only due to photoelectric absorption. In the $0.5-1.0 \mathrm{keV}$ range the high to low ratio of 1.277 can be mimicked by a change from $N_{\mathrm{H}}=1.485 \times 10^{22}$ to $1.540 \times 10^{22} \mathrm{~cm}^{-2}$, keeping all other parameters constant. The almost "grey" flux variation from 400 to $900 \mathrm{eV}$ is clearly at variance with what would be expected from photoelectric absorption by cold material with changing column density. The high to low flux ratio is significantly larger above $\sim 940 \mathrm{eV}$. Unfortunately RGS data are not sensitive enough to confirm the EPIC pn behavior. In the absence of overall spectral model, no clear explanation exists yet for this high energy bump. Remaining pile-up effects could account for part or all of the increase. However, results from the diagnostics SAS 5.3 task epatplot suggest that pile-up is not strong enough to account for all of the observed effect.

\section{Radial velocities}

Although we do not have yet any spectral model adequate for representing RGS data, the weakness of the observed spectral changes with either orbital phase or X-ray flux level offers the possibility to search for radial velocity variations, ignoring thus 

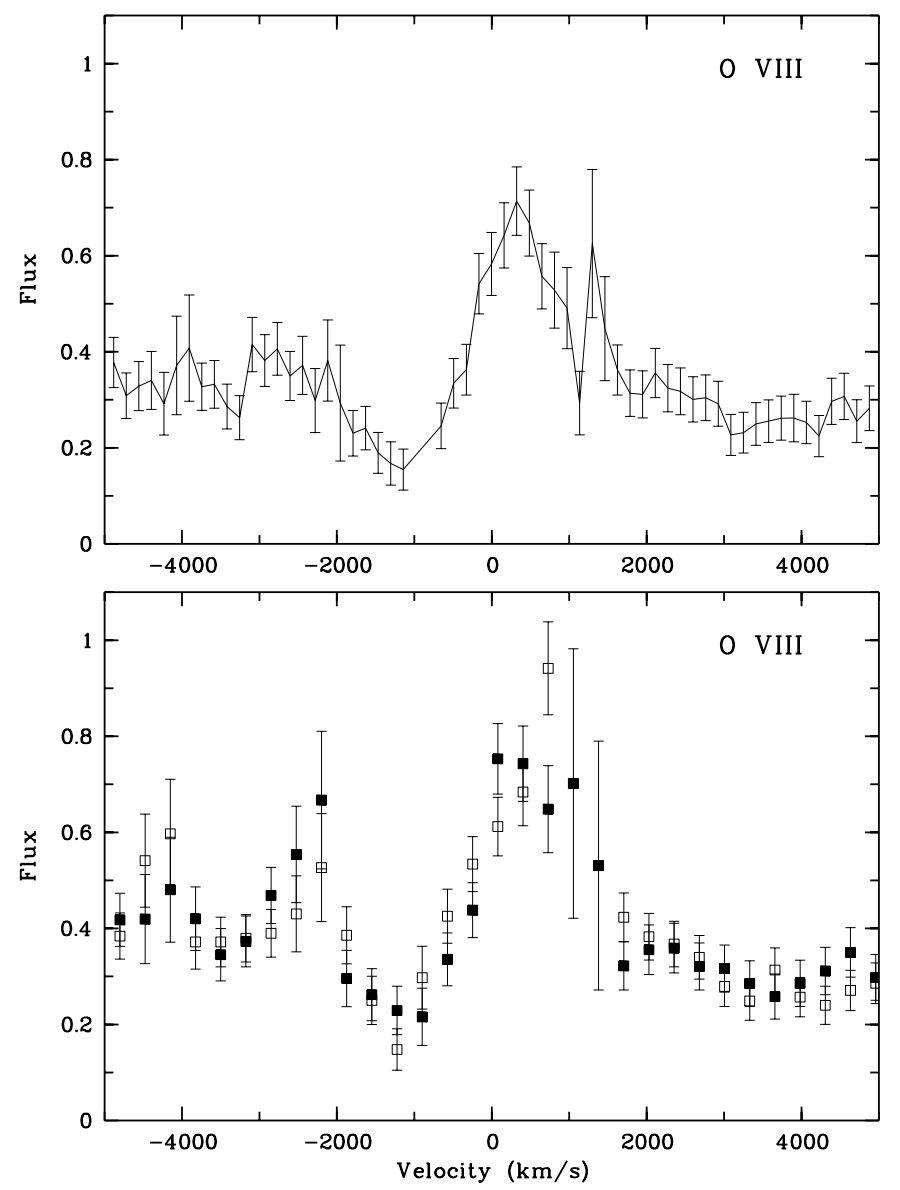

Fig. 7. Top: O viII Ly $\alpha$ P Cygni profile summing all RGS data. Bottom: low flux (open squares) and high flux (filled squares) $\mathrm{O}$ vIII Ly $\alpha$ P Cygni profiles. Low flux data have been multiplied by 1.23 to fit the mean high flux level.

the complications of the unknown underlying physics and taking advantage of the wealth of relatively sharp spectral features. The lack of identified unblended lines from the stellar atmosphere does not allow to compute absolute velocities and furthermore the RGS wavelength calibration may not be known with enough accuracy. However, comparison of the two observations obtained two days apart may allow to reveal radial velocity changes with orbital phase. For that purpose we applied a cross correlation algorithm, frequently used in optical spectroscopy and described in details in Tonry \& Davis (1979). Spectra were created using SAS task rgsfluxer for each instrument and observation. Their wavelength step of $0.0103 \AA$ samples well the instrument $F W H M$ resolution which is $\sim 0.07 \AA$ $\left(\sim 1200 \mathrm{~km} \mathrm{~s}^{-1}\right)$ in the $14-19 \AA$ range where most of the flux is present. After removing wavelength bins with high associated errors the $\log \lambda$ spectra were linearly interpolated over a velocity grid with constant steps of $15 \mathrm{~km} \mathrm{~s}^{-1}$. Absorption edges produced by cold material have much less amplitude and structures than those of emission lines from the hot atmosphere. Therefore, these features which may be partly fixed in velocity should have little influence on the amplitude of global Doppler shifts. In the wavelength range $14.18 \AA$ to $18.55 \AA$ the peak resulting from the cross-correlation of the merged RGS 1 and 2 spectra from revolutions 187 and 188 has a Gaussian

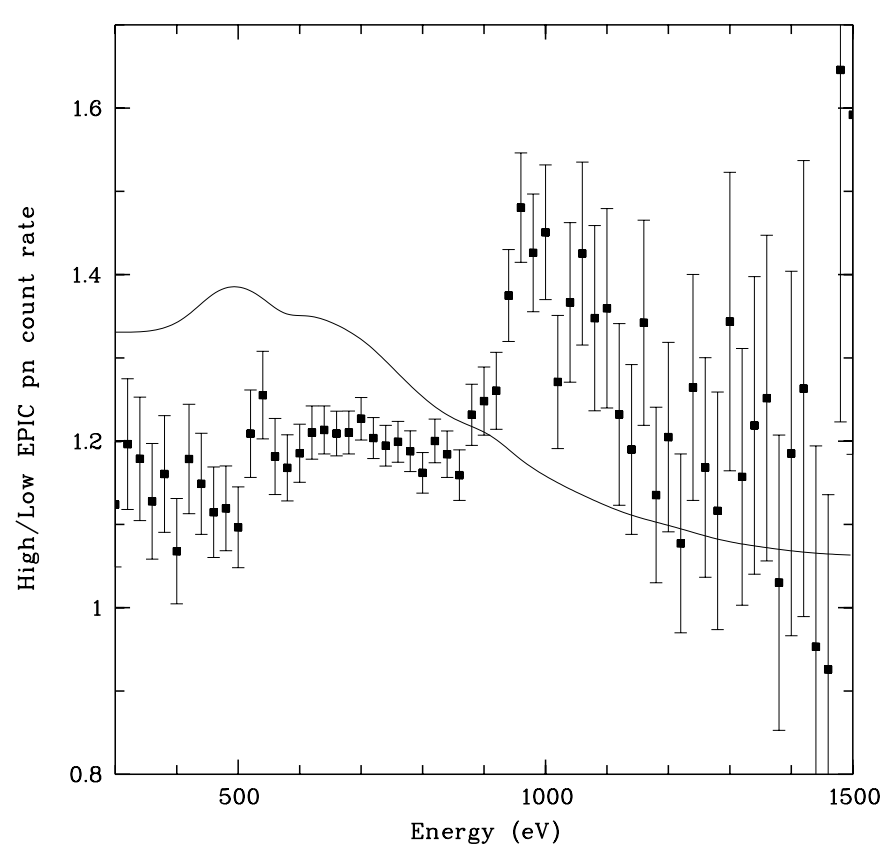

Fig. 8. High to low EPIC pn count ratio plotted versus energy. The solid line represents the spectral changes expected if intensity variations were only due to photoelectric absorption by cold plasma.

Table 2. Radial velocity shifts between revolutions 187 and 188 .

\begin{tabular}{ccc}
\hline \hline Detector & $\lambda$ range $(\AA)$ & Velocity $\left(\mathrm{km} \mathrm{s}^{-1}\right)$ \\
\hline RGS 1 + RGS 2 & $14.18-18.85$ & $173 \pm 47$ \\
RGS 1 + RGS 2 & $14.94-15.43$ & $290 \pm 93$ \\
RGS 1 + RGS 2 & $16.45-17.72$ & $164 \pm 71$ \\
RGS 1 & $14.18-18.85$ & $194 \pm 80$ \\
RGS 2 & $14.18-18.85$ & $159 \pm 67$ \\
\hline
\end{tabular}

shape and its center is displaced by $\sim 170 \mathrm{~km} \mathrm{~s}^{-1}$ (see Fig. 9 , left panel).

In order to estimate the significance of this velocity shift we generated artificial RGS spectra adding to the observed data a random value normally distributed with a sigma equal to the assigned error of the wavelength bin. In this manner we can realistically account for the varying statistical quality over the spectrum. These artificial spectra were cross-correlated applying the same procedure as the real ones and the resulting peak was fitted by a Gaussian. This process was typically repeated between 1000 and 5000 times. The distribution of the Gaussian centroids is clearly shifted whatever the wavelength interval used between $14.18 \AA$ and $18.55 \AA$ and independently of the instrument considered, R1 alone, R2 alone or R1 + R2 merged (see Fig. 9, right panel). We list in Table 2 the results of the Monte Carlo error calculation for different configurations.

Obviously, the most accurate determination is obtained by using the largest wavelength range and the two RGS detectors. However, the measured displacements are all consistent especially those derived from the completely independent RGS 1 and RGS 2 instruments. The revised ephemeris of Schmidtke \& Cowley (2001) and phase of minimum velocity read from their Fig. 4 predict for the He II $\lambda 4686$ an average velocity difference of $\sim 120$ to $140 \mathrm{~km} \mathrm{~s}^{-1}$ between observations 

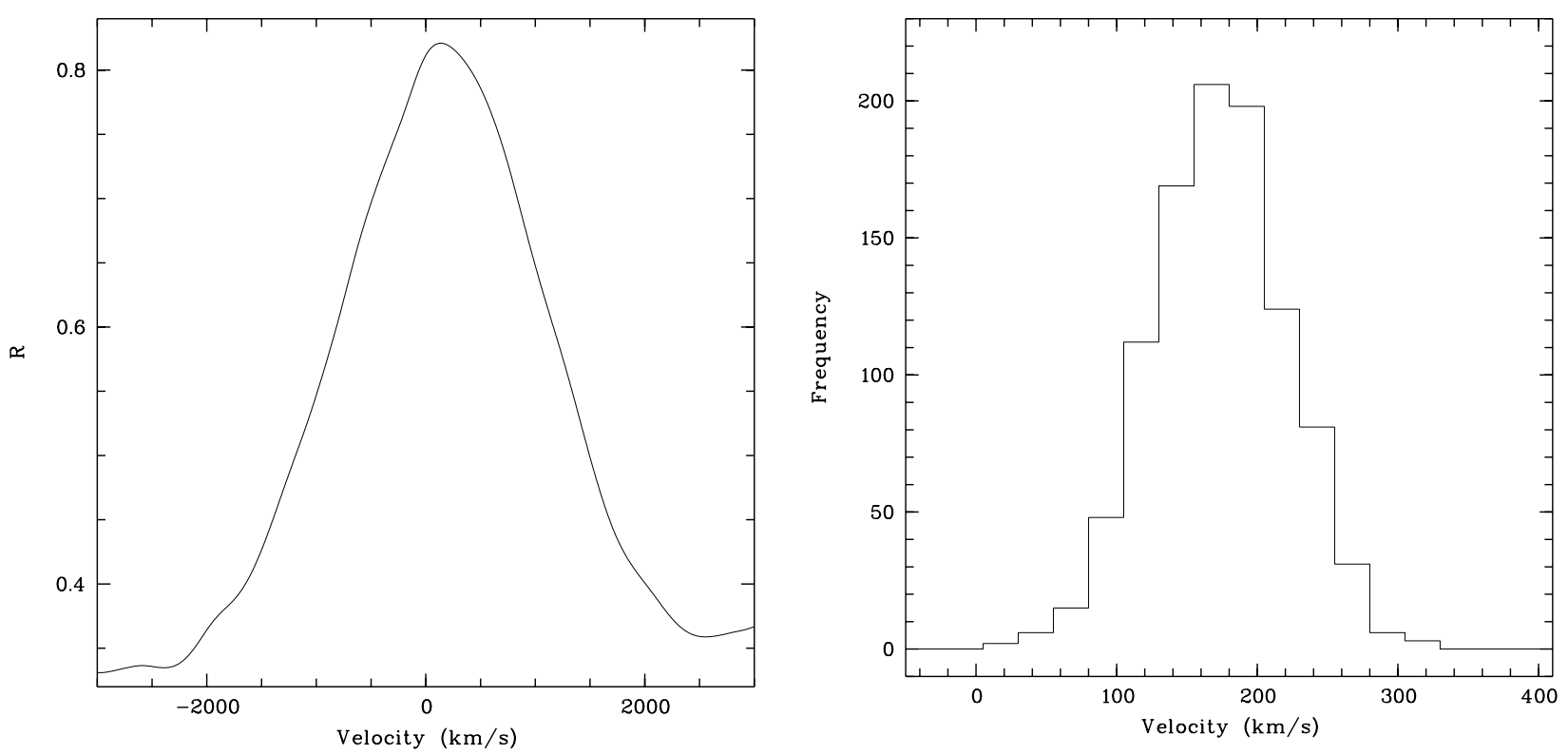

Fig. 9. Left: cross-correlation curve of the $14.18 \AA$ to $18.55 \AA$ RGS $1+2$ spectra obtained in revolutions 187 and 188 . Right: distribution of Gaussian fit centers to the cross-correlation peaks of the same randomized spectra repeated a thousand times. The mean velocity is $173 \pm$ $47 \mathrm{~km} \mathrm{~s}^{-1}$.

in revolutions $187\left(\Phi_{\text {Phot }}=0.818-0.977\right)$ and $188\left(\Phi_{\text {Phot }}=\right.$ $0.312-0.473)$. This compares well with the best RGS value of $173 \pm 47 \mathrm{~km} \mathrm{~s}^{-1}$ and gives further evidence that the velocity shift seen in the RGS is real. The sigma of the Gaussian cross correlation peak indicates an average $F W H M$ width of $\sim 3600 \mathrm{~km} \mathrm{~s}^{-1}$. This width is significantly larger than the intrinsic resolution of the RGS and suggests that line blending and/or wind effects such as seen in the Lyman $\alpha \mathrm{O}$ vIII $\mathrm{P}$ Cygni profile affect most of the spectrum.

In order to check these results we fitted with xspec Gaussian profiles on the top of a flat continuum to two of the largest and apparently weakly blended lines at $15.015 \AA$ (Fe XVII) and close to $17.6 \AA$ (Fe XVIII). We used the raw background corrected spectra and their corresponding response matrix files to fit the two RGS simultaneously. This method is in principle the most correct one since profile distortions are explicitely taken into account. Note however, that as long as spectral features do not have orbital phase dependent profiles, as it seems to be the case here, the cross correlation method applied to spectra generated by the SAS task rgsfluxer should yield correct results. Xspec fits indicate a mean displacement of $173 \pm$ $73 \mathrm{~km} \mathrm{~s}^{-1}$ comparable to that resulting from the cross correlation method. Since we only consider here two lines the significance of the velocity shift is expectedly smaller than when using all spectral features together. However, the fact that the shifts derived from xspec are comparable to those computed from the spectra created by rgsfluxer is an independent confirmation of the reality of the velocity difference between the two RGS observations.

\section{Discussion and conclusions}

XMM-Newton RGS spectra have revealed a wealth of spectral features, some of them being apparently resolved by the
RGS. Emission and also maybe absorption lines due to various iron ions are seen as well as Lyman $\alpha$ O viII. The RGS spectra confirm all the features seen in the Chandra HETGS by Bearda et al. (2002) and illustrate further the difficulty to correctly model the emission of hot white dwarf atmospheres. Clearly, more sophisticated models including wind effects are required.

The X-ray spectrum of RX J0925.7-4758 display little changes with both X-ray flux level and orbital phase. At the start of the first observation, the $\mathrm{X}$-ray source is receding with maximum positive He II velocity while two days later the source moves towards us with maximum negative He II velocities. This means that the geometrical configuration of the system is not very different between the two observations. In the absence of wake material, the column density due to the wind of the mass donor star does not change drastically and this is at first order consistent with the lack of large phase dependence of the RGS spectrum.

Very few lines show evidences for possible intensity related variations and the EPIC pn clearly indicates that absorption by cold material is not the mechanism responsible for the $\mathrm{X}$-ray modulations. Interestingly, the $0.21 \mathrm{~d}$ period for X-ray oscillations is very close to the $0.23 \mathrm{~d}$ preferred time scale reported by Schmidtke et al. (2000) in the optical light curve. The full amplitude of sine fit to the soft X-ray flux modulation at $0.21 \mathrm{~d}$ is $\sim 11 \%$ while that of the optical $V$ band modulation is between two and three times less ( 4\% Schmidtke et al. 2000). Since we do not have simultaneous optical $V$ band and X-ray observations we do not know the exact flux ratio nor phasing of the modulations. OM data are not of high enough $\mathrm{S} / \mathrm{N}$ to reveal a modulation at the level of a few percents. Observations at the Dutch telescope were too short to constrain the amplitude of a contemporaneous $0.2 \mathrm{~d}$ optical periodicity. However, such an optical to X-ray amplitude ratio is expected when optical 
emission is dominated by X-ray heating of the companion star or of the accretion disk (see e.g. van Paradijs \& McClintock 1994; Matsuoka et al. 1984). A change in the visible emitting area of the accreting white dwarf by the internal rim of the accretion disc could thus explain both the X-ray and optical $0.2 \mathrm{~d}$ modulation. Simultaneous X-ray and optical photometric observations may reveal beating effects at the $4 \mathrm{~d}$ orbital period and could thus constrain the amount of optical light resulting from X-ray heating of the mass donor star and/or of the accretion disk bulge.

The RGS spectra also confirm the existence of a strong wind clearly affecting the Lyman $\alpha \mathrm{O}$ vIII emission line and probably many other lines. The wind velocity of $\sim 2000 \mathrm{~km} \mathrm{~s}^{-1}$ is more than two times slower than the velocity of the transient jet detected by Motch (1998) at $5200 \mathrm{~km} \mathrm{~s}^{-1}$ or of that of the candidate receding $\lambda 6680.3 \AA$ component proposed by Schmidtke et al. (2000) at $5350 \mathrm{~km} \mathrm{~s}^{-1}$. In the absence of realistic model atmosphere including wind effects, it is difficult to strictly rule out the presence of weak satellite lines at $\pm 5200 \mathrm{~km} \mathrm{~s}^{-1}$ in the RGS spectrum. The autocorrelation of the RGS spectra do show some signal at similar velocity shifts. However, a more likely explanation is the presence of several iron lines with the adequate spacing in wavelength.

Amazingly, the amplitude and direction of the RGS radial velocity changes with orbital phase $\left(173 \pm 47 \mathrm{~km} \mathrm{~s}^{-1}\right)$ is fully compatible with that expected from the He II $\lambda 4686$ optical line. This observation thus supports the idea that in this system at least, the $\mathrm{He}$ II $\lambda 4686$ velocity represents the orbital motion of the X-ray source. This may be one of the first observation measuring orbital Doppler shifts in high energy spectral features from an X-ray source. Our coverage in orbital phase is not dense enough to derive the amplitude and phasing of the X-ray lines with respect to the optical $\mathrm{He}$ II line. As for the $\mathrm{H} \alpha$ line, some of the He II emission may arise from the heated hemisphere of the companion star or from the bulge of the accretion disc. This additional component may reduce the amplitude of the He II velocity change with respect to $K_{\mathrm{X}}$ and add some phase lag. A long RGS observation of RX J0925.7-4758 may thus be able to measure with relatively high accuracy the true orbital velocity of the accreting object. A reliable $K_{\mathrm{X}}$ estimate could help constraining the mass ratio and check whether the high mass transfer in RX J0925.7-4758 can be driven by thermally unstable Roche lobe overflow.

Acknowledgements. We thank E. Rol for carrying the optical observations at the Dutch telescope on La Silla and E. Janot-Pacheco for helping in the organization of the observations. We are also grateful to the referee for suggesting several improvements and to M. W. Pakull for useful discussions.

\section{References}

Bearda, H., Hartmann, W., Ebisawa, K., et al. 2002, A\&A, 385, 511

Bertin, E., \& Arnouts, S. 1996, A\&AS, 117, 393

den Herder, J. W., Brinkman, A. C., Kahn, S. M., et al. 2001, A\&A, 365, L7

Ebisawa, K., Asai, K., Mukai, et al. 1996, in Supersoft X-ray sources, ed. J. Greiner, 91

Ebisawa, K., Mukai, K., Kotani, T., et al. 2001, ApJ, 550, 1007

Hartmann, H. W., Heise, J., Kahabka, P., Motch, C., \& Parmar, A. N. 1999, A\&A, 346, 125

Iben, I. 1982, ApJ, 259, 244

Jansen, F., Lumb, D., Altieri, B., et al. 2001, A\&A, 365, L1

Lomb, N. R. 1976, Ap\&SS, 39, 447

Matsuoka, M., Mitsuda, K., Ohashi, T., et al. 1984, ApJ, 283, 774

Motch, C., Hasinger, G., \& Pietsch, W. 1994, A\&A, 284, 827

Motch, C. 1998, A\&A, 338, L13

Paerels, F., Rasmussen, A. P., Hartmann, H. W., et al. 2001, A\&A, 365, L308

Rappaport, S., Di Stefano, R., \& Smith, J. 1994, ApJ, 426, 692

Scargle, J. D. 1982, ApJ, 263, 835

Schmidtke, P. C., \& Cowley, A. P. 2001, AJ, 122, 1569

Schmidtke, P. C., Cowley, A. P., Taylor, V. A., Crampton, D., \& Hutchings, J. B. 2000, AJ, 120, 935

Tonry, J., \& Davis, M. 1979, AJ, 84, 1511

van den Heuvel, E. P. J., Bhattacharya, D., Nomoto, K., \& Rappaport, S. A. 1992, A\&A, 262, 97

van Paradijs, J., \& McClintock, J. E. 1994, A\&A, 290, 133

van Teeseling, A., \& King, A. R. 1998, A\&A, 338, 957 